\title{
Shielding effectiveness research of concrete wall based on shielding
}

\section{room method}

\author{
Wenli Liang ${ }^{1}$, Dongsheng $\mathrm{Wu}^{2}$, Changqi Zhu ${ }^{3}$, Lanlan Ping ${ }^{4}$, Wei Ding ${ }^{5}$ \\ 1,2,3,4,5 Anhui Jianzhu University No. 292 Ziyun Road, Hefei City, Anhui Province, China \\ liangwenli91@163.com, dongsheng_w@ahjzu.edu.cn, zcq199109@sina.com, \\ pinglanlan@ahjzu.edu.cn
}

Keyword: concrete, reinforced concrete, shielding effectiveness

Abstract With the rise of urbanization and the awareness of electromagnetic protection improves rapidly, more and more people pay attention to electromagnetic shielding effect of building. In this paper, a shielding room method is used to establish the model. The influence of wall thickness, dielectric constant, conductivity, radius of steel bar and steel bar density on shielding efficiency is studied. The result shows that with the increase of the wall thickness and electric conductivity, the shielding effect becomes better. And shielding efficiency has positive relationship with the radius and space of reinforcement bars.

\section{Introduction}

Along with the improvement of human life, higher standard of living and working environment is expected. People take more and more attention to the electromagnetic radiation around buildings [1]. On the one hand, there are a lot of electromagnetic radiation sources around the building [2]. On the other hand, there are also a large number of high frequency radiation source around the building. With the rapid development of mobile communication, a large number of mobile communication base stations have been installed to ensure the coverage and quality of the whole network [10,11]. In downtown, the distance between base stations is usually 200 to 300 meters [4]. Concrete is widely used in modern building construction, which has many advantages. There are two kinds of concrete structures in modern architecture: general concrete and reinforced concrete [8,9]. The concept of concrete electromagnetic shielding is refer to the effect of protecting or shielding electromagnetic wave by changing the structure and material of concrete wall, preventing the outward leakage of internal electromagnetic signals and the inward interference of external electromagnetic signals. Conceptually, the electromagnetic shielding is an obstacle to the transmission of electromagnetic waves [5], and the concrete can influence the propagation of electromagnetic waves by its electromagnetic shielding property.

In the past few years, we have used the finite difference time domain method to study the shielding effectiveness of the single layer reinforced concrete layer on the electromagnetic pulse from high altitude nuclear explosion, and analyzed the influence of the reinforced cross point welding on the shielding effectiveness [7]. In this paper, a concrete model is established by HFSS software, and the shielding room method is used to study the influence of the dielectric constant and other factors on the shielding effectiveness. 


\section{Test method for shielding performance}

While propagating in the air, the electromagnetic wave can be partly reflected at the junction of the plane due to the difference of electric parameters of the air and the shield, causing the loss of electromagnetic energy; other part continues to spread and transmit to the inside of the shield. Because of the presence of dielectric loss, the electromagnetic wave will be partly absorbed while entering the shielding material. When the electromagnetic wave propagates to the other surface of the shield body, the reflection and transmission also occur. The electromagnetic waves reflected in the internal shielding results in energy loss, called internal reflection loss. Electromagnetic shielding material achieves the shielding effect through three times wave reflection and absorption. The shielding properties of the material include wave absorbing properties and shielding properties. A window in the shielding room is arranged in the shielding room, and the signal source is arranged outside the shielding room, and the material is placed in the test window. The values of field strength are measured before and after sample installation, and get the shielding effectiveness in accordance with the definition of shielding effectiveness formula

$$
S E=20 \lg \frac{\left|E_{0}\right|}{\left|E_{1}\right|}
$$

\section{Simulation of shielding performance with ordinary concrete}

Here the ordinary concrete model size is $30 \mathrm{~cm} * 30 \mathrm{~cm} * 10 \mathrm{~cm}$. The material parameters for the model are $\mu=1, \varepsilon=8, \sigma=0.001 \mathrm{~S} / \mathrm{m}$ and transparency 0.5 . A box is built in the back of the concrete wall, and the boundary is set to Perfect E. Finally, an air layer is added at the periphery, the radiation boundary is arranged, the plane wave excitation is selected as the excitation source, and the incident direction is perpendicular to the concrete. The wave frequency is set to $900 \mathrm{MHz}$. To ensure the accuracy, the number of iteration is 10 , and the convergence error is 0.02 .

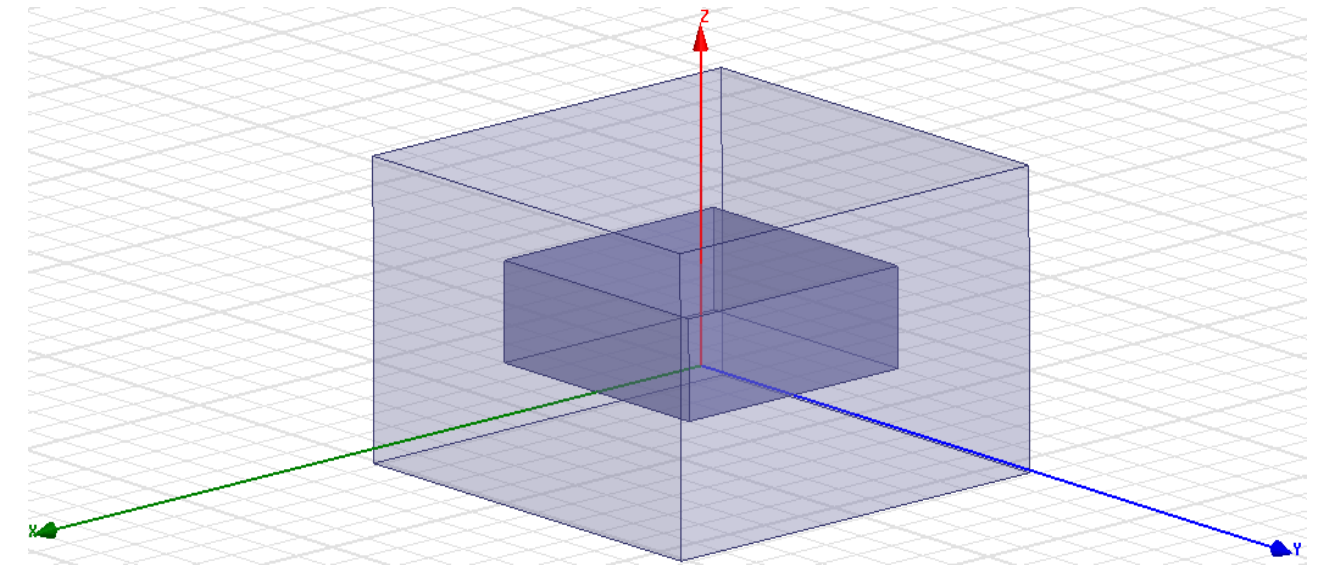

Fig.1 Test model of electromagnetic shielding effectiveness with ordinary concrete

\section{Influence of concrete thickness on shielding performance}

The concrete thickness is changed from $50 \mathrm{~mm}$ to $500 \mathrm{~mm}$ with $50 \mathrm{~mm}$ increment each time, and there are ten thickness values. With each given thickness value, the model is performed to get a shielding effectiveness value. The shielding performance is shown in Fig 1.1. 


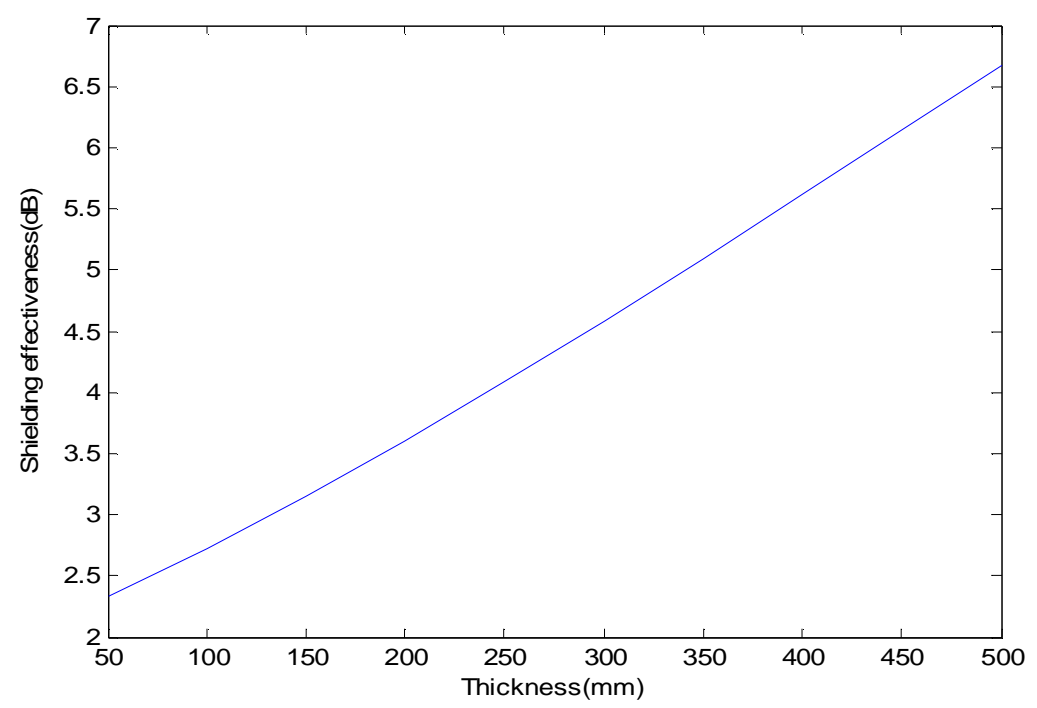

Fig.1.1 Shielding performance of concrete with different thickness

The data in the figure shows that the magnitude of the electric field is attenuated when the electromagnetic wave passes through the concrete. After passing through the 50 millimeter concrete wall, the attenuation is about 0.7 times of the original. When the concrete thickness increases, the shielding effectiveness increases, but the relationship is not linear.

\section{Influence of shielding efficiency on the dielectric constant of the concrete}

With the changes of the dielectric constant of concrete, other conditions unchanged, the shielding performance results are shown in fig.1.2.

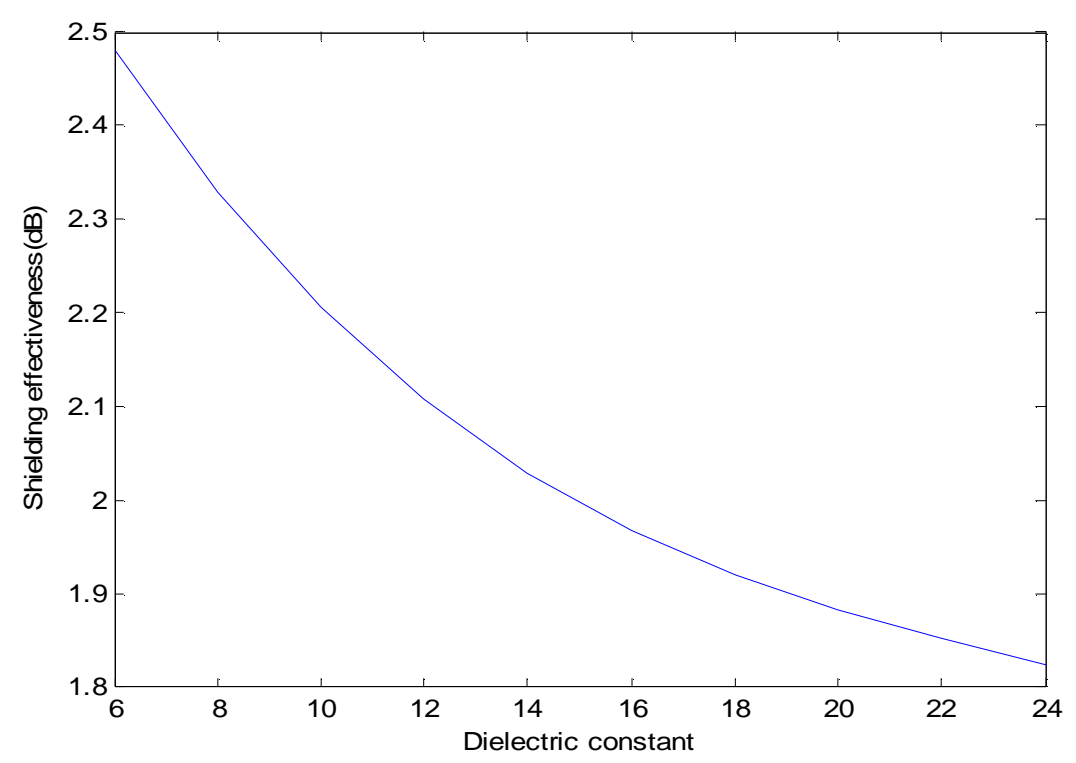

Fig.1.2 Effect of dielectric constant on shielding effectiveness of concrete

From the data in the figure, it is known that there is a decrease between dielectric constant and shielding efficiency.

Effect of electrical conductivity on shielding efficiency of concrete

With the changes conductivity of concrete and other conditions unchanged, the shielding performance is shown in fig.1.3. 


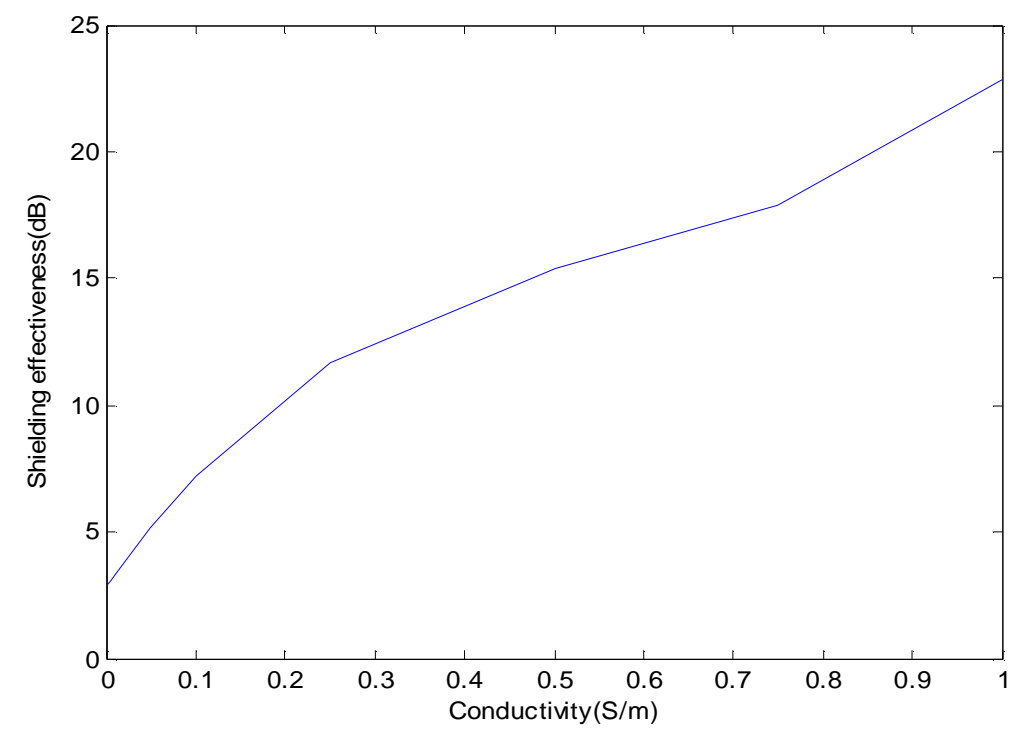

Fig.1.3 Effect of electrical conductivity on shielding effectiveness of concrete

From the fig.1.3 we can know that the greater the electrical conductivity, the better the shielding performance.

\section{Shielding performance simulation of reinforced concrete}

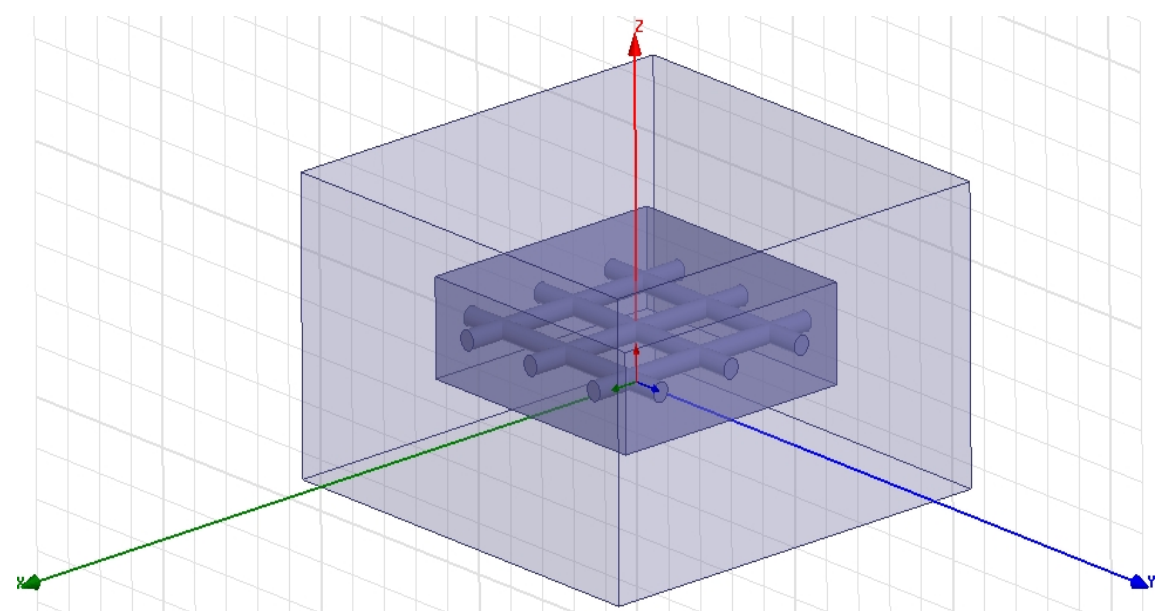

Fig.2 Test model for shielding performance of reinforced concrete wall

On the basis of ordinary concrete, the reinforced concrete model is formed by adding equal spacing to each other, as shown in figure 2. Keep other conditions unchanged without the reinforcement spacing and the diameter of reinforcing steel bar to simulate the model of reinforced concrete wall. The shielding performance is compared with the simulation results.

\section{Effect of reinforcement spacing on shielding performance}

The single layer steel mesh is added into the middle of ordinary concrete to form a reinforced concrete wall, whose radius is $6 \mathrm{~mm}$. Changes the spacing between rows or columns. The shielding performance is shown in fig.2.1. 


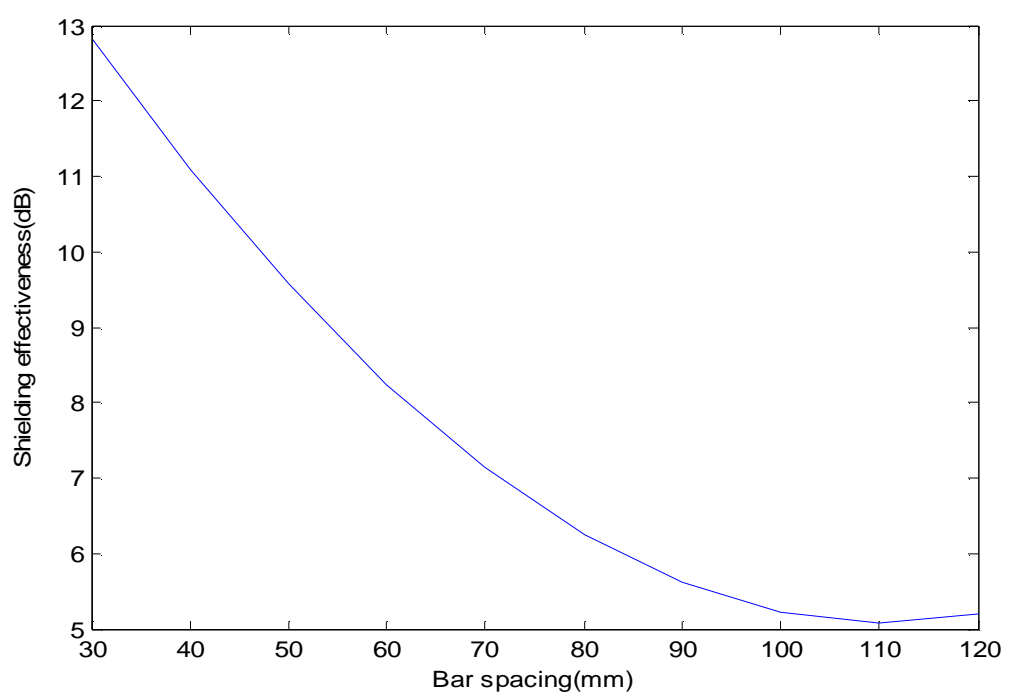

Fig.2.1 Shielding performance of reinforced concrete under different grid spacing

From the figure we can see, the smaller the mesh spacing is, the higher the shielding effectiveness of reinforced concrete is. This is because the electromagnetic wave has been reflected many times between the steel mesh and the concrete layer, so the shielding effectiveness of the electromagnetic wave is greatly increased.

\section{Effect of steel bar radius on shielding performance}

The steel mesh spacing is set to $70 \mathrm{~mm}$. Only changes the radius of reinforcement. The results are shown in fig.2.2.

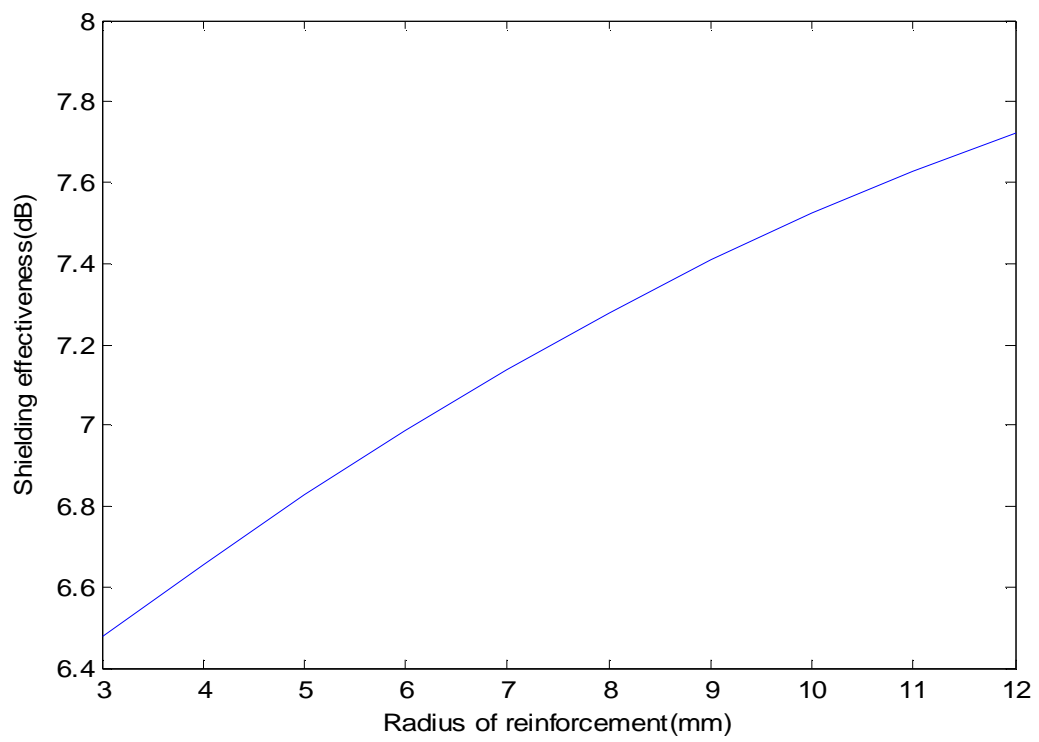

Fig.2.2 Shielding effectiveness of reinforced concrete with different radii

The relationship between the shielding performance and the radius of reinforcement is incremental. The shielding performance of the steel bar is higher than that of concrete. The lager the proportion of steel bar is, the better the overall shielding performance is.

\section{Conclusions}

A three-dimensional simulation model by HFSS was established for shielding performance test and some conclusions has been made. For ordinary concrete, its shielding effect is better when it has higher electric conductivity and lager thickness. However, the ordinary concrete is a low loss 
medium in the electromagnetic wave band which can not achieve large-scale electromagnetic shielding, so its shielding effectiveness is limited. After adding reinforcing steel bar in concrete, the shielding performance is significantly improved, and the spacing and radius of the steel bars have great influence on the shielding performance. The shielding performance will be better if the distance between the steel bars is closer and the radius is longer.

\section{Acknowledgment}

This work was supported by the National Natural Science Foundation of China (Grant No. 61471002).

\section{Reference}

[1] Meiyuan Cai. Evaluation of electromagnetic analysis and evaluation of communication base station and study of reinforced concrete shield[D]. Chongqing University .2008. pp.1-49.

[2] Sha Zong. Electromagnetic environment and electromagnetic compatibility[J]. Electronic quality. 2004.(02).pp. 33-36.

[3] Klinkcnbusch L. On the shielding effectiveness of enclosure[J]. IEEE Transactions on Electromagnetic Compatibility, 2005, 47(3):589-601.

[4] Wang Xuecheng, Jiang Weiguo, Bu Lijun. Analysis of electromagnetic radiation environment of GSM mobile communication base station [J]. Northern environment, 2004. (05). pp 33-36.

[5] Clayton,R.Paul. Introduction to Electromagnetic Compatibility[M]. Beijing: Mechanical Industry Press. 2006. first edition.

[6] Herlermann H, Koch M. Measurement of transient shielding effectiveness of shielding cabinets[J]. Advances in Radio Science, 2008, 6: 293-298.

[7] Zhou Bihua, Chen Bin, Gao Cheng, Chen Ziming. Study on electromagnetic shielding effectiveness of electromagnetic pulse in reinforced concrete and reinforced concrete[N]. Journal of radio wave science, 2005. (3). pp. 251 259.

[8] Ma Zhengping. Research on testing method of shielding effectiveness based on space field[J]. Electronics Quality, 2010 (12).pp 79-81.

[9] Li Huanran, Wen Ying. Method for measuring the shielding effectiveness[J]. Test and measurement. 2006 (06).pp45-48.

[10] Chen Xiang, Chen Yongguang, Wei Ming, Hu Xiaofeng. The material shielding effectiveness of electromagnetic pulse time domain test based on black box shielding window method[J]. High voltage technology. 2013 (03).pp. 668-674.

[11] Dalke RA, Holloway C L, McKenna P, et al. Effects of Reinforced Concrete Structures on RF Communications[J]. IEEE Transactions on Electromagnetic Compatibility, 2000,42(4) : 486 496. 